\title{
壳聚糖/有机锂皇石纳米复合材料的制备及抗菌性能研究
}

\author{
付海丽, 张雯, 张 华, 宋少波, 李伟
}

(天津工业大学 省部共建分离膜与膜过程国家重点实验室, 材料科学与工程学院, 功能纤维研究所, 天津 300387)

摘 要: 采用离子交换法将季铵盐及壳聚糖插层到锂㿝石层间，制备锂皇石基二次插层复合物。所制备的两种二次 插层复合物的层间距相比于纯的锂皇石分别增加了 $0.276 \mathrm{~nm}$ 和 $0.262 \mathrm{~nm}$ 。用大肠杆菌 $(E$. coli) 和金黄色葡萄球菌 $(S$. cereus) 为模拟体系对二次插层复合物的抗菌性进行了检验, 利用抑菌环和平板计数法测定两种二次插层复合物的 抗菌能力。抗菌结果显示，细菌与两种复合物接触 $24 \mathrm{~h}$ 后，锂皇石/十四烷基三甲基溴化铵/壳聚糖对大肠杆菌的抗 菌率可达 $100 \%$, 对 S.cereus 的抗菌率可达 $85 \%$ 以上，锂皇石/十六烷基三甲基溴化铵/壳聚糖对大肠杆菌和金黄色葡 萄球菌的抗菌率均可达 $99 \%$ 以上。采用扫描电镜法及 $\beta$-半乳糖苷酶活性考察了两种复合物对大肠杆菌和金黄色葡 萄球菌抑菌过程及机理，结果表明：复合物首先通过表面带的正电荷将表面带负电的细菌吸附到材料表面，随后 借助有机物阳离子的疏水作用，穿透细菌细胞膜，从而达到杀菌效果。

关 键 词: 有机锂㿝石; 壳聚糖; 抗菌机理; 二次插层; 纳米复合物

中图分类号: TB33 文献标识码: A

\section{Preparation and Antibacterial Activity of Chitosan/Organic Laponite Nanocomposites}

\author{
FU Hai-Li, ZHANG Wen, ZHANG Hua, SONG Shao-Bo, LI Wei
}

(State Key Laboratory of Seperation Membranes and Membrane Processes, School of Materials Science and Engineering, Tianjin Polytechnic University, Tianjin 300387, China)

\begin{abstract}
Laponite-based secondary intercalation compound was prepared by ion exchange method through intercalating quaternary ammonium salts and chitosan into the layers of hectorite. The layer spacing of the two kinds of secondary intercalation compounds were increased $0.276 \mathrm{~nm}$ and $0.262 \mathrm{~nm}$, respectively, compared to the pure laponite. The antibacterial of secondary intercalation compound was tested by Escherichia coli (E. coli) and Staphylococcus aureus (S. cereus). Antibacterial ability of the two kinds of secondary intercalation compounds were determined by using bacteriostatic ring and plate count methods. The results showed that, after being contacted with the two compounds for $24 \mathrm{~h}$, the bacteriostatic rate of the laponite/myristyl trimethyl ammonium bromide/chitosan on E. coli reached up to $100 \%$ and $85 \%$ on S.cereus. Meanwhile, the laponite/cetyl trimethyl ammonium bromide/chitosan was up to $99 \%$ on $E$. coli and $S$. cereus. The antibacterial process and mechanism on E. coli and $S$. cereus of the two kinds of compounds were investigated by scanning electron microscope and $\beta$-galactosidase activity. The results showed that the compound adsorbed bacterial through the role of mutual attraction between positive and negative charges at first, then the compound penetrated the bacterial cell membrane with the help of hy-
\end{abstract}

收稿日期: 2015-10-15; 收到修改稿日期：2015-12-25

基金项目: 国家自然科学基金(51573135); 天津市高等学校科技发展基金(20140305); 国家级大学生创新创业训练计划 (201410058012)

Nationall Natural Science Found of China (51573135); Tianjin City High School Science \& Technology Fund Planning Project (20140305); National Training Programs of Innovation and Entrepreneurship for Undergraduates (201410058012)

作者简介: 付海丽(1990-), 女, 硕士研究生. E-mail: fh1900412@126.com

通讯作者: 张 华, 教授. E-mail: hua1210@126.com; 张 雯, 讲师. E-mail: zhangwen2050@hotmail.com 
drophobic interaction of organic cation, and finally exerted the sterilization effect.

Key words: organic laponite; chitosan; antibacterial mechanism; secondary intercalation; nanocomposites

天然抗菌剂源于自然界, 高效、低毒、可降解, 是环境友好型高分子聚合物。随着环保意识的加强 及生物技术研发水平的迅速提高, 天然抗菌剂越来 越受到重视。壳聚糖(Chitosan, CS) 是一种天然碱性 多糖, 含有活性 $-\mathrm{NH}_{2}$ 和 $-\mathrm{OH}$, 具有广谱抗菌作用, 对人体无毒、无刺激性, 已被广泛用于环保、食品 和医药等领域 ${ }^{[1-3]}$ 。但是, 壳聚糖在中性介质中溶解 性差, 其抗菌性能易受酸碱度、浓度、相对分子质 量和脱乙酰度的影响, 使其应用受到一定程度的限 制 $^{[4-5]}$ 。因此, 对壳聚糖进行改性, 将其与层状硅酸 盐粘土复合制备有机-无机杂化纳米复合材料, 利 用层状硅酸盐的纳米效应和粘土片层与壳聚糖的 表、界面效应 ${ }^{[6]}$, 使壳聚糖/纳米粘土复合物具有独 特的理化性能和优良的生物活性 ${ }^{[7]}$, 可有效扩展壳 聚糖的应用领域。

锂皇石(Laponite, LAP)是三八面体 2:1 型层状 硅酸盐的一种蒙皇石族矿物 ${ }^{[8]}$, 由上下两层直径为 $25 \mathrm{~nm}$ 、厚度为 $1 \mathrm{~nm}$ 的碟形盘构成 ${ }^{[9]}$, 与其他层状 硅酸盐相比, 具有显著的膨胀性能、较大的比表面 积和阳离子交换容量等优点 ${ }^{[10]}$ 。锂皇石可通过阳离

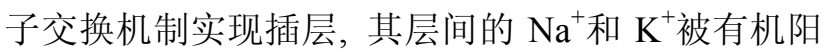
离子取代 ${ }^{[11]}$ 。锂㿝石自身可有效吸附固定细菌, 但 并不具备杀菌、抑菌作用 ${ }^{[12-13]}$, 若将具有抗菌作用 的阳离子有机物插入锂皇石片层中, 则有可能赋予 其抗菌功效。

王小英等 ${ }^{[3]}$ 将壳聚糖季铵盐与有机累托石复合 制备了纳米复合材料; 曾德芳等 ${ }^{[14]}$ 制备了改性蒙脱 石/壳聚糖复合絮凝剂。本实验采用阳离子交换法将 十四烷基三甲基溴化铵(TTAB)和十六烷基三甲基 溴化铵(CTAB) 分别插层到锂皇石(LAP)层间, 得到 两种有机改性锂㿝石, 并采用二次插层法获得既具 有吸附细菌功能又具有杀菌功能的壳聚糖/有机锂 皇石复合物, 并探讨了其抗菌机理, 为锂皇石应用 于抗菌领域提供了可能。

\section{1 实验方法}

\section{1 试剂}

TTAB 和 CTAB 均为分析纯 (天津市光复精细化 工研究所); 壳聚糖(CS, 脱乙酰度 $80 \%$ 95\%)为生 化试剂(国药集团化学试剂有限公司); 蛋白胨、酵母 浸亳粉和琼脂粉均为生化试剂(天津市光复精细化
工研究所)；2,3,5-氯化三苯基四氮唑(TTC)、邻硝基 苯 $\beta$-D-半乳吡喃糖苷 $(\mathrm{ONPG})$, 均为分析纯(上海笛 柏化学品技术有限公司); 锂皇石(LAP), 购自英国 洛克伍德助剂公司; 大肠杆菌 $(E$. coli $)$ 和金黄色葡 萄球菌( $S$. aureus) 由天津医科大学提供。

\section{2 壳聚糖/有机锂皇石的制备}

将 $2 \mathrm{~g}$ 锂皇石加入一定量的去离子水中, 配制 $2 \mathrm{wt} \%$ 的锂皇石胶状溶液，再向其中加入 $1 \mathrm{~g} \mathrm{TTAB}$, $80{ }^{\circ} \mathrm{C}$ 水浴下摚拌反应 $6 \mathrm{~h}$, (得到锂皇石/十四烷基三 甲基溴化铵(LTT)(制备机理如图 1 所示)，再按锂皇 石:壳聚糖的质量比 $1: 2$, 向反应装置中加入壳聚糖, 继续反应 $6 \mathrm{~h}$, 静置、离心、洗涤、干燥、研磨, 得 到锂㿝石/十四烷基三甲基溴化铵/壳聚糖(LTTC)。 按照前述方法分别制备锂皇石/十六烷基三甲基溴 化铵(LCT)、锂皇石/十六烷基三甲基溴化铵/壳聚糖 (LCTC)和锂㿝石/壳聚糖(LC)。

\section{3 样品表征}

采用日本 Rigaku 公司 D/MAX 2500 型 X 射线 衍射仪(XRD)测试样品层间距，使用 $\mathrm{Cu}$ 靶辐射源 $(\lambda=0.154 \mathrm{~nm}), \mathrm{Ni}$ 滤波片, 扫描范围 $4^{\circ} \sim 80^{\circ}$, 扫描 速率 $0.1 \%$, 管电压 $50 \mathrm{kV}$ ，管电流 $35 \mathrm{~mA}$ 。采用日 本日立公司 Hitachi S-4800 型扫描电镜(SEM)对样 品表面的形貌进行观察。将样品粉末分散在无水乙 醇中超声 $1 \mathrm{~h}$ 后，干燥，采用日本日立公司 H-7600 型透射电子显微镜(TEM)观察样品的结构, TEM 加 速电压 $100 \mathrm{kV}$ 、电子流速 $10 \mathrm{~mA}$ 。称取一定量的样 品分散到去离子水中, 超声 $30 \mathrm{~min}$, 采用上海中晨 数宇技术设备有限公司生产的 $\mathrm{JS} 94 \mathrm{G}+$ 型微电泳仪 测定悬浮液的 Zeta 电位。

\section{4 抗菌性能测试}

\subsection{1 抑菌环测试}

将 LTTC 和 LCTC 两种复合物压片, 采用抑菌 圈法测定两种复合物的抗菌性。无菌条件下，每组
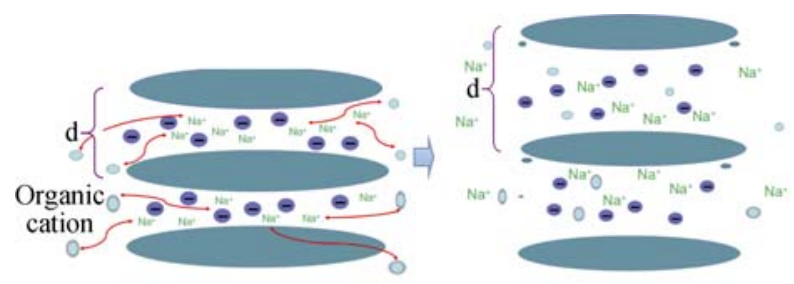

图 1 有机锂㿝石的制备机理示意图

Fig. 1 Preparation mechanism of organic laponite nanocomposites 
制作三个平行固体培养基，将大肠杆菌和金黄色葡 萄球菌于固体培养基中 $37^{\circ} \mathrm{C}$ 下培养 $24 \mathrm{~h}$ 后, 通过观 察抑菌圈的大小判断抗菌剂的抗菌能力。

\subsection{2 平板计数法测定两种复合物抗菌能力}

取 5 支已灭菌的离心管, 分别加入 $10 \mathrm{~mL}$ 培养 液、菌悬液浓度约为 $10^{7} \mathrm{cfu} / \mathrm{mL}$ 的金黄色葡萄球菌 或大肠杆菌菌悬液, 再分别向后两支离心管中加入 LTTC、LCTC、氨芐青霉素、磷酸盐缓冲液(PBS, $\mathrm{pH}=7.4$ )各 $0.1 \mathrm{~g}$, 置于 $37^{\circ} \mathrm{C}$ 摇床下培养 $24 \mathrm{~h}$ 后, 分 别逐级稀释，对不同复合物的试液，选取三个不同 浓度梯度菌液各 $0.2 \mathrm{~mL}$ 分别铺平板, 在 $37^{\circ} \mathrm{C}$ 固体培 养基下培养 $24 \mathrm{~h}$ 后, 进行平板计数, 从而得到各试 液的活菌数, 抗菌率按下式计算:

$$
\text { 抗菌率 }=\left(\frac{\text { 原菌数 }- \text { 活菌数 }}{\text { 原菌数 }}\right) \times 100 \%
$$

\subsection{3 电镜观察}

分别称取 $0.1 \mathrm{~g}$ 锂皇石与两种改性锂皇石, 再分 别加入 $10 \mathrm{~mL}$ 菌悬液浓度为 $10^{7} \mathrm{cfu} / \mathrm{mL}$ 的大肠杆菌 和金黄色葡萄球菌液体培养液, 于 $37{ }^{\circ} \mathrm{C}$ 恒温摇床 中振荡不同的时间后取出离心, 倒掉离心后的上清 液, 用 $2.5 \%$ 龙二醛固定, 再用不同浓度梯度的乙醇 溶液逐级洗脱，干燥，喷金，用 SEM 观察。

\subsubsection{TTC 显色}

取三个雉形瓶, 分别加入 $0.1 \mathrm{~mol} / \mathrm{L}$ 的葡萄糖溶 液、 $1 \mathrm{~g} / \mathrm{L}$ 的 TTC 溶液、 $0.05 \mathrm{~mol} / \mathrm{L}$ 的 $\mathrm{Tris}-\mathrm{HCl}$ 缓 冲液 $(\mathrm{pH}=8.5)$ 各 $2 \mathrm{~mL}$, 与 $1 \mathrm{~mL}$ 大肠杆菌菌液或金 黄色葡萄球菌直接混合后, 向其中两个雉形瓶中分 别加入 LTTC 和 LCTC。静置一段时间, 观察悬浊液 颜色的变化。

\subsection{5 内膜渗透性}

取三个 $100 \mathrm{~mL}$ 的雉形瓶, 向其中加入 $6 \mathrm{~mL}$ PBS 缓冲溶液 $(\mathrm{pH}=7.4)$, 一定菌浓度的菌液 $200 \mu \mathrm{L}$, $25 \mathrm{~mol} / \mathrm{L}$ ONPG $400 \mu \mathrm{L}$, 并向其中两个雉形瓶中 分别加入 $0.1 \mathrm{~g} \mathrm{LTTC}$ 和 LCTC, 置于 $37{ }^{\circ} \mathrm{C}$ 摇床中 振荡不同时间，用紫外分光光度计检测原菌液及 加入复合物后的上清液在 $420 \mathrm{~nm}$ 处吸光度随时 间的变化 ${ }^{[13]}$ 。

\section{2 结果与讨论}

\subsection{XRD 结构分析}

由图 2 可以看出, 与纯锂㿝石 $\left(2 \theta=6.77^{\circ}(001)\right.$, $d=1.334 \mathrm{~nm})$ 相比 ${ }^{[15]}$, 经过季铵盐和壳聚糖改性的 锂皇石复合物在低角度的衍射峰发生了前移, 这说 明季铵盐和壳聚糖已经成功插入锂皇石层间, 从而

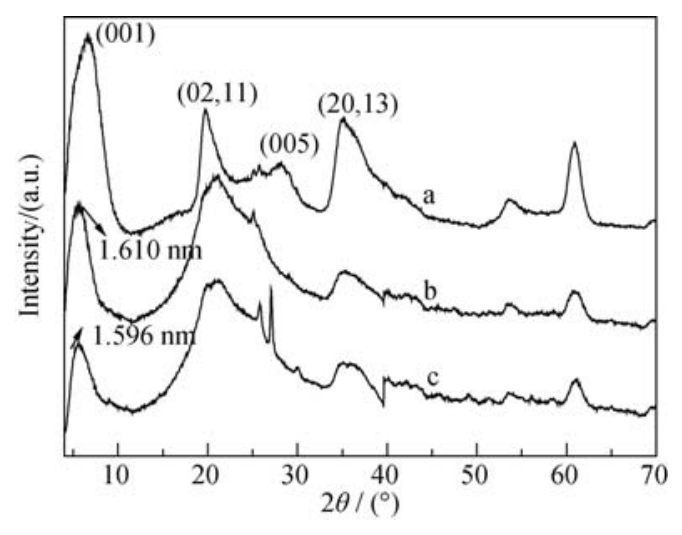

图 2 锂㿝石与壳聚糖/有机锂㿝石的 XRD 图谱

Fig. 2 XRD pattems of laponite and chitosan/modified laponite (a) LAP; (b) LTTC; (c) LCTC

形成了纳米复合材料。由 Bragg 方程计算得出, 改 性后的锂皇石复合物 LTTC 和 LCTC 层间距分别为 $1.610 \mathrm{~nm}$ 和 $1.596 \mathrm{~nm}$, 相对于纯锂皇石(001), LTTC (001)的层间距增加了 $0.276 \mathrm{~nm}, \operatorname{LCTC}(001)$ 的层间 距增加了 $0.262 \mathrm{~nm}$, 这是由于锂皇石表面壳聚糖分 子链通过离子交换反应“针固”在粘土片层上，而 有机改性后的锂㿝石与聚合物之间具有较强的作 用力 ${ }^{[16]}$, 因此更有利于壳聚糖进入锂皇石片层中。

\section{2 壳聚糖/改性锂皇石的微观形貌}

图 3 为纯锂皇石和壳聚糖/改性锂皇石的 SEM 和 TEM 照片, 由图中可看出纯的锂皇石表面呈现 比较规整平滑的状态, 在透射电镜下呈现比较均一 的颜色，而改性锂皇石呈现灰黑相间的现象，呈不 规则形状，表面有细小的颗粒吸附，表面片层无序 排列, 这与有机阳离子进入层间有关 ${ }^{[17-18]}$ 。季铵盐 和壳聚糖分子链进入锂皇石片层间, 导致层与层间 膨胀, 固体微观形态结构蓬松, 层间距增大, 分子 间作用力减小, 这些结果和上述 XRD 结果相一致。
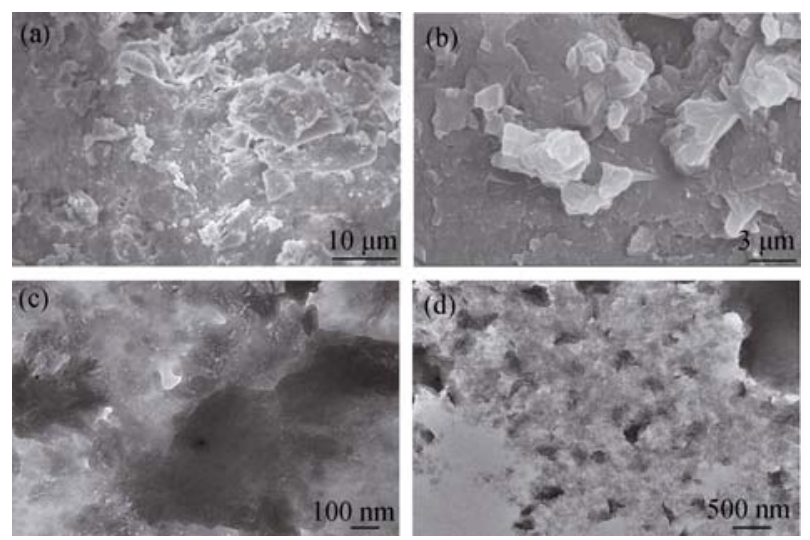

图 3 锂㿝石(a, c) 和壳聚糖/改性锂㿝石(b, d)的 $\operatorname{SEM}(a, b)$ 和 $\operatorname{TEM}(\mathrm{c}, \mathrm{d})$ 照片

Fig. 3 SEM $(a, b)$ and TEM $(c, d)$ images of laponite and chitosan/modified laponite 


\section{3 抗菌性能测定}

有机物的存在能够改变锂皇石层间微环境物化 性能和层间几何空间, 直接影响有机锂皇石的化学 性质和物理应用 ${ }^{[16]}$ 。由图 4 可见, LTT、LCT、LC 对大肠杆菌和金黄色葡萄球菌的抑菌圈均不及 LTTC 和 LCTC 的抑菌圈明显, 表明二次插层复合可提高 复合物的抗菌性能。且季铵盐和壳聚糖可以起到协 同抗菌的作用。其中两种二次插层复合物对金黄色 葡萄球菌的抑菌圈直径大于大肠杆菌的抑菌圈直径, 这是因为金黄色葡萄球菌表面所带的负电荷高于大 肠杆菌, 表面带正电荷的二次插层复合物对金黄色 葡萄球菌的吸附作用更强。

从表 1 数据看出, 两种二次插层复合物与两种 细菌接触 $24 \mathrm{~h}$ 后, LTTC 对大肠杆菌的抑菌率可达 $100 \%$, 对金黄色葡萄球菌的抗菌率可达 $85 \%$ 以上, 而 LCTC 对两者的抗菌率均可达 99\%以上, 说明二 次插层复合物具有更好的抗菌作用。

\section{4 壳聚糖/有机锂皇石抗菌过程观察}

图 5(a)为正常的大肠杆菌照片, 图 5(a')为正常 金黄色葡萄球菌, 分别呈现棒状和球形, 细菌边界 清晰，还可看到有正在分裂的细菌。图 5(b)和图 5(b') 分别为大肠杆菌和金黄色葡萄球菌与复合物接触 $4 \mathrm{~h}$
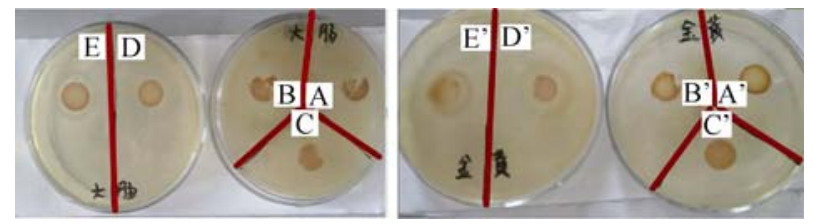

图 4 改性锂㿝石对大肠杆菌(A-E)和金黄色葡萄球菌(A'-E') 的抑菌圈

Fig. 4 Antimicrobial activity of LTT (A, A'), LCT (B, B'), LC (C, C'), LTTC (D, D') and LCTC (E, E') against E. coli and $S$. aureus

的扫描电镜照片，可看出部分菌体开始出现聚集及 凹陷，且边界逐渐模糊。图 5(c)和图 5(c') 分别为大 肠杆菌和金黄色葡萄球菌与复合物接触 $10 \mathrm{~h}$ 的扫描 电镜照片，菌体已发生严重的变形和皱缩，且聚集 现象更明显，部分菌体已失去原有形态，出现严重 的粘连, 有细胞碎片的出现。随着接触时间延长至 $24 \mathrm{~h}$ 时, 如图 5(d)和 5(d') 可以看到菌体已基本失去 原有形态, 呈现出严重的皱缩变形及粘连现象, 边 界不明显。出现以上现象的主要原因是锂㿝石表面 吸附有机阳离子后，使其由亲水性转变成亲油性 [19]，从而与细菌细胞壁的亲脂成分，例如脂蛋白、 脂多糖、磷脂等成分产生疏水作用 ${ }^{[20]}$, 扰乱细胞膜 的正常活动, 从而导致细菌变形死亡。

表 1 LTTC 和 LCTC 的抑菌率

Table 1 Bactericidal ratio of LTTC and LCTC

\begin{tabular}{cccccc}
\hline \multirow{2}{*}{ Samples } & \multicolumn{2}{c}{ E. coli } & & \multicolumn{2}{c}{ S.cereus } \\
\cline { 2 - 3 } \cline { 5 - 6 } & $\begin{array}{c}\text { Recovered bacteria count } \\
/\left(\mathrm{cfu} \cdot \mathrm{mL}^{-1}\right)\end{array}$ & $\begin{array}{c}\text { Antimicrobial ratio } \\
/ \%\end{array}$ & & $\begin{array}{c}\text { Recovered bacteria count } \\
/\left(\mathrm{cfu} \cdot \mathrm{mL}^{-1}\right)\end{array}$ & $\begin{array}{c}\text { Antimicrobial ratio } \\
/ \%\end{array}$ \\
\hline Blank & $2.59 \times 10^{8}$ & 0 & $3.40 \times 10^{9}$ & 0 \\
LTTC & 0 & 100.0 & $5.00 \times 10^{8}$ & 85.3 \\
LCTC & $1.10 \times 10^{4}$ & 99.9 & $1.00 \times 10^{4}$ & 99.9 \\
Positive control & $2.30 \times 10^{4}$ & 99.9 & $1.00 \times 10^{3}$ & 99.9 \\
Negative control & $3.30 \times 10^{8}$ & 0 & $2.86 \times 10^{9}$ & 15.9 \\
\hline
\end{tabular}
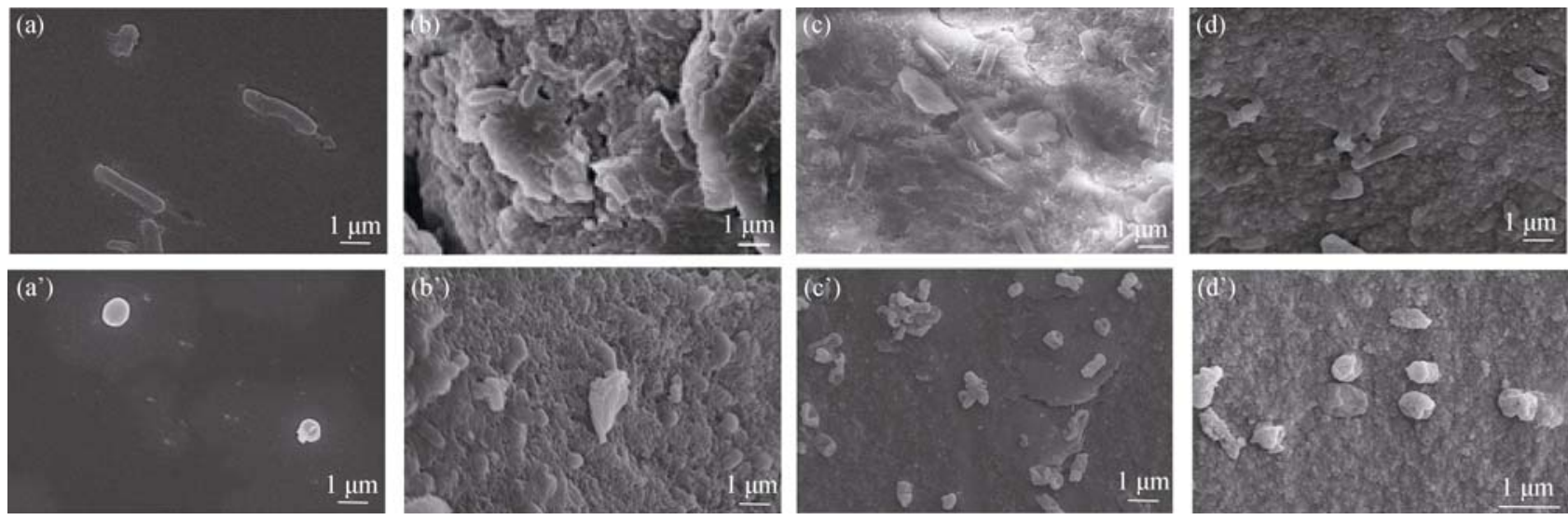

图 5 大肠杆菌( $\mathrm{a} \sim \mathrm{d})$ 和金黄色葡萄球菌( $\left.\mathrm{a}^{\prime} \sim \mathrm{d}^{\prime}\right)$ 处理后的壳聚糖/有机锂皇石的 SEM 照片

Fig. 5 SEM images of chitosan/modified laponite after treatment with E.coli (a-d) and S.cereus (a'-d') 


\subsection{Zeta 电位分析}

为研究季铵盐及壳聚糖的插入对锂皇石表面带 电性的影响, 分别对锂皇石及壳聚糖/改性锂皇石做 Zeta 电位测试, 并对测试结果求方差及平均值, 测 试结果如表 2 所示。从表 2 可看出纯锂皇石带负电, 而经壳聚糖和季铵盐改性的锂皇石表面则具有正的 Zeta 电位值, 这意味着改性锂皇石表面的正电荷得 到加强, 证明了季铵盐及壳聚糖已成功引入。同时, 改性锂皇石表面所带的正电荷使其更有利于吸附表 面带负电荷的细菌, 从而提高抗菌剂吸附细菌及杀 菌的能力。

\section{6 抗菌机理研究}

图 6(a)和 6(a') 分别是金黄色葡萄球菌和大肠杆 菌菌液, 悬浊液显红色, 表明菌体具有活性, 可以 将 TTC 转化为 $\mathrm{TF}$ 。图 6(b) 是金黄色葡萄球菌液和 LTTC 的混合液, 图 6(b') 是大肠杆菌菌液和 LTTC 的混合液, 悬浊液呈无色, 复合物颗粒略显红色, 表明菌体大部分已被破坏。图 6(c)是金黄色葡萄球 菌菌液和 LCTC 的混合液, 图 6(c') 是大肠杆菌菌液

表 2 LAP、LTTC 和 LCTC 的 Zeta 电位值 Table 2 Zeta potential of LAP, LTTC and LCTC ( $n=3)$

\begin{tabular}{ccc}
\hline Sample & Static dissipative & Zeta potential $/ \mathrm{mV}$ \\
\hline LAP & - & $-27.6 \pm 4.8$ \\
LTTC & + & $43.0 \pm 1.6$ \\
LCTC & + & $82.3 \pm 2.1$ \\
\hline
\end{tabular}
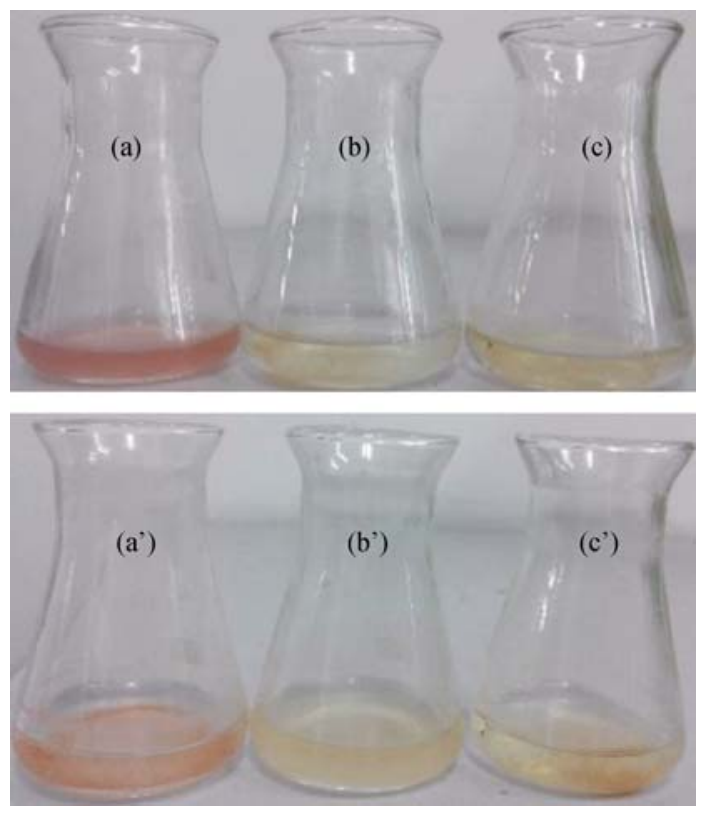

图 6 不同样品对大肠杆菌和金黄色葡萄球菌的抗菌效果 比较

Fig. 6 Comparison of S.cereus (a-c) and E. coli (a'-c') activity under different samples

(a) Blank; (b) LTTC; (c) LCTC
和 LCTC 的混合液, 此时悬浊液呈无色, 复合物颗 粒部分显红色，表明菌体大部分被破坏。

图 7 显示, 随着时间的推移, 未加抗菌剂的菌 液在 $420 \mathrm{~nm}$ 处的吸光度基本不随时间而变化，说明 细菌的内膜没有受到破坏，而加入两种二次插层复 合物的菌液在 $420 \mathrm{~nm}$ 处的吸光度随着时间的延长 不断增加, 且 LTTC 在 $420 \mathrm{~nm}$ 处的吸光度基本都大 于 LCTC，说明前者对大肠杆菌的抑菌作用优于后 者。由此推测, 两种抗菌剂的作用位点在细菌的细 胞膜上, 且抗菌性随着时间的延长而增强。

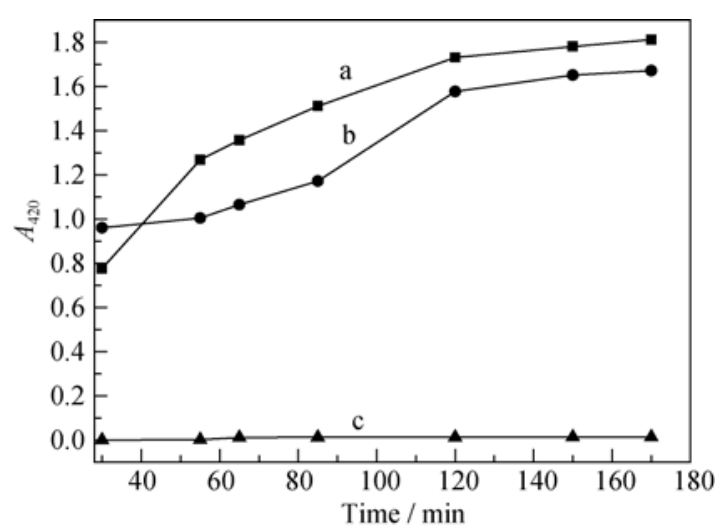

图 7 经过壳聚糖/改性锂㿝石处理后内膜渗透性变化

Fig. 7 Change of permeability of bacterial inner membrane under the condition of chitosan/modified laponite

(a) LTTC; (b)LCTC; (c) Blank

\section{3 结论}

实验成功合成了两种锂皇石基二次插层复合物, 有机阳离子的加入使锂皇石表面由带负电荷转变为 带正电荷，并且两种复合物对大肠杆菌和金黄色葡 萄球菌均具有很强的抑制和杀菌作用。样品与细菌 接触 $24 \mathrm{~h}$ 后, LTTC 对大肠杆菌的抗菌率可达 $100 \%$, 对金黄色葡萄球菌的抗菌率可达 $85 \%$ 以上，LCTC 对大肠杆菌和金黄色葡萄球菌的抗菌率均可达 $99 \%$ 以上。壳聚糖/有机锂皇石的抗菌机理为，复合物通 过表面所带的正电荷将表面带负电的细菌吸附到材 料表面，随后借助有机物阳离子的疏水作用，穿透 细菌细胞膜, 从而达到杀菌效果。

\section{参考文献:}

[1] LU Y H, CHENG D H, LU S, et al. Preparation of quaternary ammonium salt of chitosan nanoparticles and their textile properties on Antheraea pernyi silk modification. Textile Research Journal, 2014, 84(19): 2115-2124.

[2] LIM S H, HUDSON S M. Synthesis and antimicrobial activity of a 
water-soluble chitosan derivative with a fiber-reactive group. Carbohydrate Research, 2004, 339: 313-319.

[3] WANG XIAO-YING, DU YU-MIN, SUN RUN-CANG, et al. Antimicrobial activity of quaternized chitosan/organic rectorite nanocomposite. J. Inorg. Mater., 2009, 24(6): 1236-1242.

[4] MAO Z W, MA L, YAN J, et al. The gene transfection efficiency of thermoresponsive N, N, N-thimethyl chitosan chloride-g-poly (N-ispropylacrylamide) copolymer. Biomaterials, 2007, 28: 4488-4500.

[5] LI WEI, LI MING-CHUN, XIN MEI-HUA, et al. Synthesis and antibacterial properties of furfutal modified chitosan derivatives. Chemical Industry and Engineering Progress, 2014, 33(4): 966-970.

[6] SHU ZHONG-JUN, LIU XIAO-HUI, QI ZONG-XIONG. Research of polymer/clay nanocomposites. China Plastics, 2000, 14(3): $12-18$.

[7] WANG XIAO-YING, DU YU-MIN, SUN RUN-CANG, et al. Chitosan-based layered silicate nanocomposites. Process in Chemistry, 2009, 21(7/8): 1507-1514.

[8] HEIKE P, BOSBACH D, PANAK P J, et al. Eu(III) copercipitation with the trioctahedral clay mineral hectorith. Clays and Clay Minerals, 2006, 54(1): 45-46.

[9] GUO YING WANG, DINA MACIEL, YILUN WU, et al. Amphiphilic polymer-mediated formation of laponite-based nanohybrids with robust stability and $\mathrm{pH}$ sensitivity for anticancer drug delivery. ACS Appl. Mater. Interfaces, 2014, 6: 16687-16695.

[10] GHADIRI M, CHRZANOWSKI W, ROHANIZADEH R. Antibiotic eluting clay mineral (Laponite) for wound healing application: an in vitro study. J. Mater. Sci.: Mater. Med., 2004(25): 2513-2526.

[11] LI Z, CHANG P H, JIANG W T, et al. Mechanism of methylene blue removal from water by swelling clays. Chem. Eng. J., 2011, 168: $1193-1200$.
[12] XI XI, ZHEN WEI-JUN, BIAN SHEN-ZHEN. In-situ intercalative polymerization of poly(lactic acid) / HTCC-saponite nanocomposites and their properties characterization. Chemical Journal of Chinese Universities, 2015, 36(3): 559-567.

[13] HE YONG, GAO ZHAO-FEN, XIN-YAN. Crystallization behavior of poly(-lactide). Chemical Journal of Chinese Universities, 2006, 27(4): 745-748.

[14] ZENG DE-FANG, CHENG JIE. Treatment of papermaking waste water using modified montmorillonite chitosan flocculant. Environmental Protection of Chemical Industry, 2009, 29(2): 144-146.

[15] WANG S G, ZHENG F Y, HUANG Y P, et al. Encapsulation of amoxicillin within laponite-doped poly(lactic-co-glycolic acid) nanofibers: preparation, characterization, and antibacterial activity. ACS Applied Materials \& Interfaces, 2012, 4: 6393-6401.

[16] NIE JIAN-HUA, ZHENG DA-FENG, PI PI-HUI, et al. Research progress of hectorite modified by organic compounds. Chemical Industry and Eegineering Progress, 2008, 27(12): 1903-1916.

[17] BASKARALINGAM P, PULIKESI M, RAMAMURTHI V, et al. Modified hectorites and adsorption studies of a reactive dye . Appl Clay Sci., 2007, 37(1/2): 207-214.

[18] XU EN-HUI, ZHENG QIANG, WANG CAI-HONG, et al. Studies on the preparation and properties of PA6 /organic hectorite nanocomposites. Actapolymerica Sinica, 2015, 5: 515-523.

[19] MESSERSMITH P B, GIANNELIS E P. Synthesis and barrier properties of poly(e-caprolactone)-layered silicate nanocomposites. Journal of Applied Polymer Science Part A: Polym. Chem., 1995, 33(10): 47-57.

[20] HOFFMAN J D, MILLER R L. Kinetic of crystallization from the melt and chain folding in polyethylene fractions revisited: theory and experiment. Polymer, 1997, 38(13): 3151-3212. 